\title{
Functional connectivity after hemispherectomy
}

\author{
Yvonne Höller ${ }^{1}$, Viviana Versace ${ }^{2,3}$, Eugen Trinka ${ }^{4,5,6}$, Raffaele Nardone $^{7,8,9}$ \\ ${ }^{1}$ Faculty of Psychology, University of Akureyri, Akureyri, Iceland; ${ }^{2}$ Department of Neurorehabilitation, Hospital of Vipiteno, Vipiteno, Italy; \\ ${ }^{3}$ Research Unit for Neurorehabilitation South Tyrol, Bolzano, Italy; ${ }^{4}$ Department of Neurology, Paracelsus Medical University Salzburg, Salzburg, \\ Austria; ${ }^{5}$ Centre for Cognitive Neurosciences Salzburg, Salzburg, Austria; ${ }^{6}$ University for Medical Informatics and Health Technology, UMIT, \\ Hall in Tirol, Austria; ${ }^{7}$ Franz Tappeiner Hospital, Merano, Italy; ${ }^{8}$ Spinal Cord Injury and Tissue Regeneration Center Salzburg, Salzburg, Austria; \\ ${ }^{9}$ Department of Neurology, Paracelsus Medical University Salzburg, Salzburg, Austria
}

Correspondence to: Raffaele Nardone. Franz Tappeiner Hospital, Merano, Italy; Spinal Cord Injury and Tissue Regeneration Center Salzburg, Salzburg, Austria; Department of Neurology, Paracelsus Medical University Salzburg, Salzburg, Austria. Email: raffaele.nardone@sabes.it.

Provenance and Peer Review: This article was commissioned by the Editorial Office, Quantitative Imaging in Medicine and Surgery. The article did not undergo external peer review.

Comment on: Kliemann D, Adolphs R, Tyszka JM, Fischl B, Yeo BTT, Nair R, Dubois J, Paul LK. Intrinsic functional connectivity of the brain in adults with a single cerebral hemisphere. Cell Rep 2019;29:2398-407.e4.

Submitted Mar 08, 2020. Accepted for publication Mar 20, 2020.

doi: 10.21037/qims.2020.03.17

View this article at: http://dx.doi.org/10.21037/qims.2020.03.17

What happens to brain networks after removal of a cerebral hemisphere? This question was addressed in a recently published fMRI study by Kliemann and colleagues (1). The authors examined a sample of six high functioning individuals at the age of 20-31 years, who underwent anatomical hemispherectomy between birth and the age of 10. Investigation of these chronic cases has revealed important insights into long-term adaptation of brain networks to hemispherectomy. The impressive result was that the interhemispheric connectivity in brains with only one hemisphere is strikingly similar to the functional network organization in brains with two hemispheres. In particular, the interaction between attention network and default mode network was comparable between the group after hemispherectomy and a healthy control group. Most interestingly, global efficiency of networks in patients was increased compared to controls whereas modularity was not altered. This result leaves room for speculations. It can be hypothesized that there is an intrinsic plan for these networks, which evolves regardless of whether there are two or only one hemisphere in the brain. We may even claim that this result provides further evidence for the redundant organization of the brain. Kliemann et al. (1) also suggested that more typical connectivity is likely a marker for more successful compensation and, thus, intact cognitive abilities.

Cerebral reorganization occurs remarkably early and adaptively promotes functional recovery after hemispherectomy (2). Accordingly, there exist reports about successful recovery after hemispherectomy both in children (3) and adults (4,5). Hemispherectomy is an effective therapy in medically intractable epilepsy with a seizure-free rate of $73 \%$ across 56 studies (6). The finding that brain networks return to a quasi-normal state (1) may considerably increase the positive attitude towards this highly invasive, and technically challenging surgical technique.

Brain networks, such as the default mode network, became a prominent issue after their introduction in fMRI research (7). However, examination of brain networks extends beyond this research method. Fast propagating networks can be more adequately characterized by methods with a higher time resolution, such as electroencephalography (EEG) or magnetoencephalography (MEG). It was shown in two patients with disorders of consciousness (DOC) after functional hemispherectomy that results from multiple modalities such as resting state 
${ }^{18} \mathrm{~F}$-fluorodeoxyglucose positron emission tomography (FDG-PET), fMRI and EEG are convergent in the demonstration of a functionally preserved hemisphere (8). The authors hypothesized that the fact that dysfunctional hemodynamic fMRI resting state connectivity analyses were matched by the more direct metabolic FDG-PET studies provides evidence for their origin being of neuronal nature rather than originating from noise from the scanner, movement, respiratory, or cardiac artifacts. An additional challenge in patients with severe injuries is the deformation of the brain, which render group-level analyses, as well as comparisons with healthy controls, challenging if not impossible.

In the study of Bruno et al. (8), the EEG showed lateralized delta dysrhythmia, which was consistent with metabolic PET and hemodynamic fMRI results. Indeed, cerebral lesions or dysfunctions are often seen as slowed activity in the EEG (9). Bruno et al. (8) also employed diffusion tensor imaging (DTI) analyses in order to determine whether the observed functional abnormalities were caused by axonal or cortical damages. This method takes advantage of the molecular movement of water within axons, i.e., white matter tracts, and is therefore able to show minimal changes in structural connections that may not be detectable with standard MRI (10). The clear advantage of DTI is that-in contrast to functional connectivity as applied in the study of Kliemann et al. (1)—a tracked connection with DTI exists physically, whereas functional connectivity can be intuitively understood as a statistical similarity of activity, but does not necessarily involve a structural connection between the studied regions. In severe traumatic brain injury, DTI provides a reliable tool to quantify the degree of injury $(11,12)$ and allows to monitor recovery (13). However, it was shown that functional connectivity is usually based on underlying structural anatomy. Horn and colleagues compared results from DTI and functional connectivity measures in healthy subjects and suggested that structural connectivity usually leads to functional connectivity while the inverse relation is not always straightforward (14). A potential confounder for functional connectivity is the situation where there is little or no structural connectivity between two regions A and $\mathrm{B}$, but a third region $\mathrm{C}$ is physically connected to both $\mathrm{A}$ and $\mathrm{B}$ and mimics therefore a direct functional link (15). An interesting case of a patient in unresponsive wakefulness syndrome (UWS) showed functional impairment in the default mode network, but preservation of structural connectivity in terms of white matter. Most interestingly, the patient recovered and regained consciousness (16). This finding suggests that different levels of impairment have also diagnostic and prognostic value-an issue that should be further explored.

Notably, consciousness is preserved with only one hemisphere, and structural or functional connectivity can predict functional outcome to some extent. However, in the absence of a complete understanding of the neural correlates of consciousness, no conclusions can be drawn with respect to a potential residual phenomenological awareness in patients who appear to be unresponsive but present with a functionally preserved, isolated unilateral hemisphere function.

Another field of research that deserves attention, when discussing research on subjects with functional or effective hemispherectomy, is the examination of split-brain patients. Research on split-brain patients has revealed differences in cognitive processing between the two disconnected hemispheres (17). Unlike the situation of chronic hemispherectomy after removal of a hemisphere, such as in the cases presented by Kliemann et al. (1) where functions are located in only one hemisphere, in cases of UWS with functional hemispherectomy, such as those described by Bruno et al., it is still possible that the lack of a clinical proof of consciousness may be due to a deficiency of some sensory, core (18), or primary (19) functions of consciousness in one hemisphere. This possibility is supported by observations of residual right-lateralized hemispheric function on FDG-PET, fMRI, and EEG measurements (8).

Animal studies also enable to explore functional and/ or structural networks after hemispherectomy. However, neurophysiological, behavioral and functional imaging data indicate that functional rearrangement in human patients is much more complex than what can be observed in animal models. Moreover, in preclinical studies hemispherectomy is generally the first ever lesion, from which the animal should recover, while in cases of medically required hemispherectomy in humans, an extensive lesion or a pathological process exists already before the interventionmaking the intervention necessary. Therefore, in humans, abnormal brain function precedes hemispherectomy by many years. It was repeatedly shown that the neural reorganization begins prior to surgery and is mediated by extensive functional rearrangement and rewiring of the contralesional hemisphere (20). In view of the rapid reorganization after removal of the lesioned hemisphere, the "plastic role" of functional reorganization cannot be mapped 1:1 to improved functional performance. 
When studying reorganization and recovery after hemispherectomy, the distinction between crossed and uncrossed fibers is crucial. Ipsilateral corticospinal pathways descending from the contralesional hemisphere remain functional until around 10 years of age (20). It is quite straightforward by use of neuroimaging and neuromodulation techniques to study cortical reshaping after brain injury, but plasticity of ascending and descending neuronal pathway is less accessible. Causal relationships are often inferred statistically from EEG, MEG, or fMRI but actually, these methods provide only a statistical model of causality, no proof. In contrast, transcranial magnetic stimulation (TMS) can establish causality by creating transient lesions and by introducing an external source of activity where the distribution or the activity and impact of the stimulation can be measured electrically and behaviorally. Ipsilateral motor evoked potentials can be useful to study the function of ipsilateral corticospinal connections. This technique requires higher stimulation intensities, but the evoked potentials have also longer latencies and different cortical map representations. The ipsilateral corticospinal tract was found to gain strength after lesions occurring in adulthood, because of new functional demands $(21,22)$. A study by Holloway et al. suggests that when brain damage is acquired in adulthood, the cortico-reticulospinal pathways may contribute strongly to ipsilateral motor control (23). The authors reported evoked responses that were elicited through the stimulation of the primary motor cortex (M1) and premotor cortex (PMC). The interesting finding was that these responses appear to be a result of two distinct pathways that could both serve ipsilateral function, since both of them contain fast-conducting fibers. These two pathways are the ipsilateral corticospinal tract, which originates in M1, and the cortico-reticulospinal pathway, which originates from the PMC.

In relation to this, studies in patients after extensive unilateral brain injuries demonstrated that the contralesional hemisphere can take over responsibilities of the affected side, even in adult patients (20). This process involves changes of pathways between contralesional cortex and subcortical regions as well as spinal cord segments, which are functionally and anatomically connected to the affected side of the body. Indeed, the size of the injury matters: small lesions lead primarily to ipsilesional rearrangement, but larger lesions induce functional reorganization in the contralesional hemisphere (23).

A more common condition than hemispherectomy is an extensive unilateral stroke. This condition is often followed by engagement of homologous motor areas in the contralesional hemisphere to serve the control of ipsilateral limbs early after onset, thus showing that transcallosal interactions contribute to functional recovery, but significant upregulation of the contralateral hemisphere correlates with poor outcome (24). Mapping this finding back to the situation of patients after hemispherectomy, we should take the shift of activity to the contralesional hemisphere into consideration. However, in contrast to stroke, the hemisphere that is resected in hemispherectomy is not intact, so that inhibitory transcallosal interactions might reflect a maladaptive process before hemispherectomy; the lesioned hemisphere may exert abnormal inhibition toward the contralesional one (20). Indeed, existing data on asymmetric activity and, thus, on a disbalance between excitation and inhibition are the basis of treatments aiming at reducing the activity of the contralesional hemisphere, and thus at rebalancing the activity of the two hemispheres, in particular by means of inhibitory repetitive TMS (25).

Although reports on patients in chronic states such as UWS without recovery support the expectation that large brain lesions usually lead to severe impairment, studies in patients after hemispherectomy illustrate the remarkable resilience of the brain to extensive unilateral lesions. The high level of functioning of patients after hemispherectomy is explained by findings showing complex anatomical and functional rearrangement. In view of this growing body of evidence, clinical implications arise. Postlesional examination of functional and structural networks could inform rehabilitation strategies aiming to improve functional recovery following unilateral hemispheric lesions (20) and also to predict functional outcome (25). However, the functional examination of brain networks after "functional" or "structural" hemispherectomy is not limited to fMRI, as shown by multimodal studies. Considering the financial deficit of health systems in many western countries and limited availability of MRI in smaller institutions and areas with poorly equipped clinics, quantitative EEG methods should also be further developed to explore functional plasticity and connectivity after hemispherectomy.

\section{Acknowledgments}

Funding: Dr. Höller reports research was funded by the Austrian Science Fund (FWF) (T 798-B27) and the Research Fund of Paracelsus Medical University (PMU- 
FFF) (A-16/02/021-HÖL).

\section{Footnote}

Conflicts of Interest: All authors have completed the ICMJE uniform disclosure form (available at http://dx.doi. org/10.21037/qims.2020.03.17). ET has acted as a paid consultant to Eisai, EVER Neuro Pharma, Biogen Idec, Medtronics, Bial, and UCB and has received speakers' honoraria from Bial, Eisai, Boehringer Ingelheim, Biogen, Newbridge, Novartis, and UCB Pharma in the past 3 years. ET has received research funding from UCB Pharma, Biogen, Novartis, Bayer, Eisai, Red Bull, Merck, the European Union, Austrian Science Fund (FWF), and Bundesministerium für Wissenschaft und Forschung. ET is also part of the investigators planning the ESET Trial and a member of the Task Force on Classification of Status Epilepticus of the International League Against Epilepsy. The other authors have no conflicts of interest to declare.

Open Access Statement: This is an Open Access article distributed in accordance with the Creative Commons Attribution-NonCommercial-NoDerivs 4.0 International License (CC BY-NC-ND 4.0), which permits the noncommercial replication and distribution of the article with the strict proviso that no changes or edits are made and the original work is properly cited (including links to both the formal publication through the relevant DOI and the license). See: https://creativecommons.org/licenses/by-nc-nd/4.0/.

\section{References}

1. Kliemann D, Adolphs R, Tyszka JM, Fischl B, Yeo BTT, Nair R, Dubois J, Paul LK. Intrinsic functional connectivity of the brain in adults with a single cerebral hemisphere. Cell Rep 2019;29:2398-407.e4.

2. Sebastianelli L, Versace V, Taylor A, Brigo F, Nothdurfter W, Saltuari L, Trinka E, Nardone R. Functional reorganization after hemispherectomy in humans and animal models: What can we learn about the brain's resilience to extensive unilateral lesions? Brain Res Bull 2017;131:156-67.

3. Griessenauer CJ, Salam S, Hendrix P, Patel DM, Tubbs RS, Blount JP, Winkler PA. Hemispherectomy for treatment of refractory epilepsy in the pediatric age group: a systematic review. J Neurosurg Pediatr 2015;15:34-44.

4. Schmeiser B, Zentner J, Steinhoff BJ, Schulze-Bonhage A, Kogias E, Wendling AS, Hammen T. Functional hemispherectomy is safe and effective in adult patients with epilepsy. Epilepsy Behav 2017;77:19-25.

5. Schusse CM, Smith K, Drees C. Outcomes after hemispherectomy in adult patients with intractable epilepsy: institutional experience and systematic review of the literature. J Neurosurg 2018;128:853-61.

6. Hu WH, Zhang C, Zhang K, Shao XQ, Zhang JG. Hemispheric surgery for refractory epilepsy: a systematic review and meta-analysis with emphasis on seizure predictors and outcomes. J Neurosurg 2016;124:952-61.

7. Fox MD, Raichle ME. Spontaneous fluctuations in brain activity observed with functional magnetic resonance imaging. Nature Reviews Neuroscience 2007;8:700-11.

8. Bruno MA, Fernández-Espejo D, Lehembre R, Tshibanda K, Vanhaudenhuyse A, Gosseries O, Lommers E, Napolitani N, Noirhomme Q, Boly M, Papa M, Owen A, Maquet P, Laureys S, Soddu A. Multimodal neuroimaging in patients with disorders of consciousness showing "functional hemispherectomy". Prog Brain Res 2011;193:323-33.

9. Brenner RP. The interpretation of the EEG in stupor and coma. Neurologist 2005;11:271-84.

10. Le Bihan D. Looking into the functional architecture of the brain with diffusion MRI. Nat Rev Neurosci 2003;4:469-80.

11. Benson RR, Meda SA, Vasudevan S, Kou Z, Govindarajan KA, Hanks RA, Millis SR, Makki M, Latif Z, Coplin W, Meythaler J, Haacke EM. Global white matter analysis of diffusion tensor images is predictive of injury severity in traumatic brain injury. J Neurotrauma 2007;24:446-59.

12. Huisman TA, Schwamm LH, Schaefer PW, Koroshetz WJ, Shetty-Alva N, Ozsunar Y, Wu O, Sorensen AG. Diffusion tensor imaging as potential biomarker of white matter injury in diffuse axonal injury. AJNR Am J Neuroradiol 2004;25:370-6.

13. Sidaros A, Engberg AW, Sidaros K, Liptrot MG, Herning M., Petersen P, Paulson OB, Jernigan TL, Rostrup E. Diffusion tensor imaging during recovery from severe traumatic brain injury and relation to clinical outcome: A longitudinal study. Brain 2008;131:559-72.

14. Horn A, Ostwald D, Reisert M, Blankenburg F. The structural-functional connectome and the default mode network of the human brain. Neuroimage 2014;102:142-51.

15. Damoiseaux JS, Greicius MD. Greater than the sum of its parts: A review of studies combining structural connectivity and resting-state functional connectivity. Brain Struct Funct 2009;213:525-33. 
16. Fernández-Espejo D, Bekinschtein T, Monti MM, Pickard JD, Junque C, Coleman MR, Owen AM.

Diffusion weighted imaging distinguishes the vegetative state from the minimally conscious state. Neuroimage 2011;54:103-12.

17. Gazzaniga MS. Cerebral specialization and interhemispheric communication: Does the corpus callosum enable the human condition? Brain 2000;123:1293-326.

18. Damasio AR. Investigating the biology of consciousness. Philos Trans R Soc Lond B Biol Sci 1998;353:1879-82.

19. Edelman GM. Wider than the sky: The phenomenal gift of consciousness. New Haven and London: Yale University Press, 2004.

20. Sebastianelli L, Saltuari L, Nardone R. How the brain can rewire itself after an injury: the lesson from hemispherectomy. Neural Regen Res 2017;12:1426-7.

21. Ziemann U, Ishii K, Borgheresi A, Yaseen Z, Battaglia F, Hallett M, Cincotta M, Wassermann EM. Dissociation of the pathways mediating ipsilateral and contralateral

Cite this article as: Höller Y, Versace V, Trinka E, Nardone R. Functional connectivity after hemispherectomy. Quant Imaging Med Surg 2020;10(5):1174-1178. doi: 10.21037/qims.2020.03.17 motor-evoked potentials in human hand and arm muscles. J Physiol 1999;518:895-906.

22. Nardone R, Langthaler PB, Orioli A, Versace V, Scarano GI, Brigo F, Saltuari L, Carnicelli L, Trinka E, Sebastianelli L. Ipsilateral motor evoked potentials in a patient with unihemispheric cortical atrophy due to Rasmussen encephalitis. Neural Regen Res 2019;14;1025-8.

23. Holloway V, Gadian DG, Vargha-Khadem F, Porter DA, Boyd SG, Connelly A. The reorganization of sensorimotor function in children after hemispherectomy. A functional MRI and somatosensory evoked potential study. Brain 2000;123:2432-44.

24. Staudt M, Grodd W, Gerloff C, Erb M, Stitz J, KrägelohMann I. Two types of ipsilateral reorganization in congenital hemiparesis: a TMS and fMRI study. Brain 2002;125:2222-37.

25. Bradnam LV, Stinear CM, Byblow WD. Ipsilateral motor pathways after stroke: implications for non-invasive brain stimulation. Front Hum Neurosci 2013;7:184. 